\title{
Divergent pathologies and treatment options for diabetic neuropathies
}

\author{
Matti Uusitupa ${ }^{1} \cdot$ Leo Niskanen ${ }^{2} \cdot$ Tomi Laitinen $^{3}$ \\ Received: 30 April 2020 / Accepted: 8 June 2020 / Published online: 13 July 2020 \\ (C) Springer-Verlag GmbH Germany, part of Springer Nature 2020
}

Keywords Diabetic autonomic neuropathy $\cdot$ Diabetic somatic neuropathy $\cdot$ Glycaemic control $\cdot$ Pathophysiology

\author{
Abbreviation \\ DPS Finnish Diabetes Prevention Study
}

To the Editor: In an excellent review on polyneuropathy in patients with diabetes, Callaghan et al [1] question the significance of hyperglycaemia in the prevention and treatment of neuropathy in individuals with type 2 diabetes. They concluded that intensive glycaemic control only marginally improved neuropathy in type 2 diabetes cohorts. This view was based on the lack of definitive evidence from a small number of intervention studies in individuals with type 2 diabetes, and the conclusions depended heavily on the Action to Control Cardiovascular Risk in Diabetes (ACCORD) study, which accounted for $70.6 \%$ of the evidence [2]. There are many reasons for this quite modest level of evidence, as also discussed by the authors. Furthermore, we should keep in mind that the aetiology of type 2 diabetes is heterogeneous based on recent genetic studies, and that the phenotype is also variable, ranging from relative insulin deficiency to marked insulin resistance, commonly associated with obesity. In line with the heterogenous nature of the disease, in 1995 we published a 10-year follow-up study on the development of neuropathy in newly diagnosed individuals with type 2 diabe-

Matti Uusitupa

matti.uusitupa@uef.fi

1 Institute of Public Health and Clinical Nutrition, University of Eastern Finland, P.O. Box 70211, Kuopio, Finland

2 Department of Internal Medicine, Päijät-Häme Central Hospital, Lahti, Finland

3 Department of Clinical Physiology and Nuclear Medicine, University of Eastern Finland and Kuopio University Hospital, Kuopio, Finland tes [3]. The diagnosis was based on both clinical symptoms and signs, and neurophysiological measurements during follow up, making this study unique in the field. There was a remarkable increase in the development of neuropathy in diabetic individuals from baseline $(8.3 \%)$ to 10 -year examination $(41.9 \%)$. In the non-diabetic control group, the respective figures were $2.1 \%$ and $5.8 \%$. Besides hyperglycaemia, low fasting and postprandial insulin and C-peptide values were, independently of glucose values, associated with the risk of peripheral somatic neuropathy. We suggested that, in individuals with type 2 diabetes, good glycaemic control should be maintained from the time of diagnosis. Patients who develop marked insulin deficiency should be treated with insulin therapy to correct hyperglycaemia in order to prevent neuropathy, due to the known beneficial effects of insulin on hyperglycaemia and a potential role of insulin deficiency, per se in the pathophysiology of diabetic neuropathy [3]. It is of note that, on average, metabolic control in individuals with type 2 diabetes was quite poor in 1990s as compared with present day, which may explain the observed clear relationship between glucose values and the long-term risk of neuropathy in our study [3].

Callaghan et al barely mention diabetic autonomic neuropathy as a part of the diabetic neuropathy concept [1]. Autonomic neuropathy may have different pathologies to peripheral neuropathy. We consider autonomic neuropathy underdiagnosed concerning its multiple clinical manifestations. Furthermore, cardiac autonomic neuropathy is associated with an increased cardiovascular morbidity and mortality risk in individuals with diabetes. In our study on newly diagnosed patients with type 2 diabetes [4], both hyperglycaemia and high insulin values were associated with the risk of autonomic neuropathy, strongly suggesting that insulin resistance plays a role in the pathogenesis of autonomic nervous dysfunction among obese individuals with type 2 diabetes. We suggested divergent pathologies for autonomic and 
peripheral somatic neuropathies in these diabetic participants $[4,5]$. This view was supported by our study in individuals with impaired glucose tolerance as part of the Finnish Diabetes Prevention Study (DPS). In this cross-sectional follow-up study, age, weight, BMI, waist circumference, serum triacylglycerols and triacylglycerol/HDL-cholesterol ratio were associated with autonomic neuropathy. In contrast, fasting or $2 \mathrm{~h}$ glucose values during an OGTT were not significantly associated with autonomic nervous dysfunction in this subgroup analysis of the DPS [6]. Regarding the data on serum triacylglycerols, these results are in line with the notion that lipid abnormalities related to elevated triacylglycerols may contribute to the pathogenesis of neuropathy, as discussed in the review by Callaghan and colleagues [1].

Altogether, these results have important implications in practice, and individually tailored treatment options for the management of hyperglycaemia and lipid disorders in patients with type 2 diabetes are needed. Insulin and other glucoselowering therapies should be used accordingly in patients with type 2 diabetes in order to prevent the development of late complications of hyperglycaemia, including neuropathies. As for somatic and autonomic nervous dysfunction in obesity-related type 2 diabetes, weight reduction and other measures to correct insulin resistance (e.g. physical activity and healthy diet) form the basis for the prevention and treatment of the disease and its complications. It is of note that in early phases of diabetes, autonomic nervous dysfunction may be reversible after intensified (lifestyle) intervention [7, 8].

Authors' relationships and activities The authors declare that there are no relationships or activities that might bias, or be perceived to bias, their work.

Contribution statement MU drafted the manuscript and LN and TL commented on it and provided additional published data to complete the letter. Each author approved the submitted manuscript. MU is the guarantor of this work and takes full responsibility for the work as a whole, including access to data and the decision to submit and publish the manuscript.

\section{References}

1. Callaghan BC, Gallagher G, Fridman V, Feldman EL (2020) Diabetic neuropathy: what does the future hold? Diabetologia 63: 891-897. https://doi.org/10.1007/s00125-020-05085-9

2. Callaghan BC, Little AA, Feldman EL, Hughes RA (2012) Enhanced glucose control for preventing and treating diabetic neuropathy. Cochrane Database Syst Rev (6):CD007543. https:// doi.org/10.1002/14651858.CD007543.pub2

3. Partanen J, Niskanen L, Lehtinen J, Mervaala E, Siitonen O, Uusitupa M (1995) Natural history of peripheral neuropathy in patients with non-insulin dependent diabetes mellitus. N Engl J Med 333:89-94. https://doi.org/10.1056/NEJM199507133330203

4. Töyry JP, Niskanen LK, Mäntysaari MJ, Länsimies EA, Uusitupa MI (1996) Occurrence, predictors, and clinical significance of autonomic neuropathy in NIDDM. Ten-year follow-up from the diagnosis. Diabetes 45(3):308-315. https://doi.org/10.2337/diab.45.3.308

5. Töyry JP, Partanen JV, Niskanen LK, Länsimies EA, Uusitupa MI (1997) Divergent development of autonomic and peripheral somatic neuropathies in NIDDM. Diabetologia 40(8):953-958. https://doi. org $/ 10.1007 / \mathrm{s} 001250050773$

6. Laitinen T, Lindström J, Eriksson J et al (2011) Cardiovascular autonomic dysfunction is associated with central obesity in persons with impaired glucose tolerance. Diabet Med 28:699-704. https://doi.org/ 10.1111/j.1464-5491.2011.03278.x

7. Vanninen E, Uusitupa M, Länsimies E, Siitonen O, Laitinen J (1993) Effect of metabolic control on autonomic function in obese patients with newly diagnosed type 2 diabetes. Diabet Med 10(1):66-73. https://doi.org/10.1111/j.1464-5491.1993.tb01999.x

8. Rosengård-Bärlund M, Bernardi L, Fagerudd J et al (2009) Early autonomic dysfunction in type 1 diabetes: a reversible disorder? Diabetologia 52(6):1164-1172. https://doi.org/10.1007/s00125009-1340-9

Publisher's note Springer Nature remains neutral with regard to jurisdictional claims in published maps and institutional affiliations. 